\title{
2 ADHS und umschriebene Entwicklungsstörungen
}

Waldemar von Suchodoletz

\subsection{Umschriebene Entwicklungsstörungen}

Zu den umschriebenen bzw. spezifischen Entwicklungsstörungen werden nach der Internationalen Klassifikation psychischer Störungen der WHO (ICD10) umschriebene Störungen des Sprechens und der Sprache (F8o), umschriebene Entwicklungsstörungen schulischer Fertigkeiten (F81), umschriebene Störungen motorischer Funktionen (F82) und kombinierte umschriebene Entwicklungsstörungen (F83) gerechnet (WHO 2005). Für alle Entwicklungsstörungen gilt, dass die entsprechende Funktion primär betroffen und im Gegensatz zu anderen Krankheitsbildern der Verlauf stetig ist, ohne dass Phasen einer plötzlichen Verbesserung oder Verschlechterung zu beobachten sind. Charakteristisch ist zudem eine familiäre Häufung und Jungen sind öfter als Mädchen betroffen. Auch Kinder mit umschriebenen Entwicklungsstörungen zeigen deutliche Lernfortschritte, die allerdings in den betroffenen Funktionen langsamer ausfallen als dies bei unauffällig entwickelten Kindern der Fall ist.

Nach der ICD-1o sind umschriebene Sprech- und Sprachentwicklungsstörungen dadurch gekennzeichnet, dass bei einem altersgerechten Kommunikationsbedürfnis die Sprech- bzw. Sprachfertigkeiten eines Kindes außerhalb der Variationsbreite der Norm liegen und bekannte Erkrankungen oder Störungen nicht Ursache der Behinderung des Spracherwerbs sind. Sprachstörungen bedingt durch eine Intelligenzminderung, eine hirnorganische Erkrankung, eine Hörstörung, durch emotionale oder andere psychiatrische Erkrankungen, durch falsche Sprachvorbilder oder durch eine unzureichende Anregung durch die 
Umwelt werden nicht zu den umschriebenen Sprachentwicklungsstörungen gerechnet.

Umschriebene Sprech- und Sprachentwicklungsstörungen werden eingeteilt in Artikulationsstörungen sowie expressive und rezeptive Sprachstörungen. Artikulationsstörungen (Lautbildungsstörung, Stammeln, Dyslalie) gehen im Gegensatz zu Sprachstörungen nicht gehäuft mit einem ADHS oder anderen psychischen Störungen einher und bleiben deshalb im Folgenden unberücksichtigt. Expressive Sprachentwicklungsstörungen (F8o.1) sind dadurch gekennzeichnet, dass die Sprachproduktion außerhalb der Norm liegt, während das Sprachverständnis allenfalls geringfügig beeinträchtigt ist. Bei der rezeptiven Form (F8o.2) hingegen finden sich deutliche Defizite im Bereich des Sprachverständnisses meist verbunden mit solchen in der Sprachproduktion. Bei beiden Subtypen sind zusätzliche Lautbildungsstörungen die Regel.

Die Symptomatik einer Sprachentwicklungsstörung ist vom Alter des Kindes abhängig. Manche Kinder fallen bereits im Säuglingsalter durch ein vermindertes Lallen auf. Im zweiten Lebensjahr sind ein verspätetes Erlernen der ersten Wörter und eine verzögerte Entwicklung des aktiven und passiven Wortschatzes charakteristisch. Im dritten Lebensjahr stehen eine verminderte Äußerungslänge und ein weitgehendes Fehlen syntaktischer Strukturen im Mittelpunkt der sprachlichen Auffälligkeiten. Im Kindergarten- und Vorschulalter haben die Kinder besondere Schwierigkeiten bei der Bildung und dem Verständnis grammatischer Wortformen und Satzstrukturen. Im Schulalter wird die Spontansprache weitgehend fehlerfrei. Die Kinder sprechen in einfachen und kurzen Sätzen und vermeiden kompliziertere grammatische Strukturen. Probleme werden erst bei höheren Anforderungen deutlich. Den Kindern fällt es schwer, Geschichten folgerichtig zu erzählen, übertragene Bedeutungen und Mehrdeutigkeiten zu verstehen und sich schriftlich kohärent mitzuteilen. Diese Schwierigkeiten bleiben oft bis ins Erwachsenenalter hinein bestehen, werden aber erst bei besonderen Anforderungen oder einer gezielten Überprüfung deutlich. Weitere häufig zu beobachtende Sprachauffälligkeiten sind ein geringer Wortschatz sowie Wortfindungs- und Lautbildungsstörungen. Viele Kinder, bei denen auch noch im Einschulungsalter eine Sprachentwicklungsstörung nachweisbar ist, haben im Schulalter eine Lese-Rechtschreibstörung.

Die klinische Bedeutung von Sprachentwicklungsstörungen ergibt sich aus langfristigen Auswirkungen auf die sozialen Entwicklungschancen und die Persönlichkeitsentwicklung. Sind Sprachstörungen noch im Vorschulalter zu beobachten, dann ist mit einer Wahrscheinlichkeit von 70-80\% mit Sprachauffälligkeiten bis ins Jugend- und Erwachsenenalter hinein zu rechnen. $\mathrm{Zu}$ sätzlich beeinträchtigen eine Lese-Rechtschreibstörung (50-80\%), abfallende IQ-Werte (ca. 25\%) und emotionale bzw. Verhaltensstörungen (ca. 50\%) die Entwicklungschancen dieser Kinder. Insgesamt ist die Prognose bei einer Artikulationsstörung am besten und bei einer rezeptiven Sprachstörung am schlechtesten (Übersicht v. Suchodoletz 2004). 
Lese-Rechtschreibstörungen werden in der ICD-10 zu den umschriebenen Entwicklungsstörungen schulischer Fertigkeiten gerechnet. $\mathrm{Zu}$ dieser Kategorie zählen weiterhin die isolierte Rechtschreibstörung und die Rechenstörung. Am häufigsten und ausführlichsten untersucht ist die Lese-Rechtschreibstörung, auf die im Folgenden schwerpunktmäßig eingegangen werden soll.

Die Lese-Rechtschreibstörung (F81.o) wird definiert als umschriebene Beeinträchtigung der Entwicklung der Lese- und in der Regel damit verbunden der Rechtschreibfähigkeiten, die nicht durch eine Intelligenzminderung, unzureichende Lernbedingungen, unkorrigierte Seh- oder Hörstörungen, ausgeprägte neurologische Defizite oder emotionale Störungen bedingt ist. Bei der isolierten Rechtschreibstörung (F81.1) fehlen Hinweise auf eine Leseschwäche.

Im Mittelpunkt der Symptomatik stehen Schwierigkeiten beim Erwerb des Lesens und der Rechtschreibung. Den Kindern fällt es zu Beginn des Deutschunterrichtes schwer, das Alphabet zu erlernen und Buchstaben korrekt zu benennen. Später unterlaufen ihnen beim Lesen zahlreiche Fehler. Sie lassen Buchstaben und Wortteile aus, verdrehen Teile des Wortes, ersetzen Wörter oder Wortteile und fügen neue hinzu. Die Kinder lesen extrem langsam, verlieren die Zeile und die Betonung entspricht nicht der Phrasenstruktur des Satzes. Das Leseverständnis ist eingeschränkt, sodass die Kinder das Gelesene nicht wiedergeben können.

Vergleichbare Fehler treten bei der Rechtschreibung auf. Anfangs sind es Auslassungen, Einfügungen, Verdrehungen sowie Reihenfolgeumstellungen von Buchstaben und Wortteilen bis zu Wortentstellungen, später dann eine Nichtbeachtung von Rechtschreibregeln, wie Fehler in der Croß- und Kleinschreibung oder Dehnungsfehler. Verstöße gegen eine lautgetreue Schreibweise werden als Phonemfehler, solche gegen eine regelhafte Abweichung von der lautgetreuen Schreibweise als Regelfehler und solche gegen Abweichungen von Rechtschreibregeln als Speicherfehler bezeichnet.

Die klinische Relevanz einer Lese-Rechtschreibstörung ergibt sich nicht nur daraus, dass Lese- und Rechtschreibprobleme bis ins Erwachsenenalter hinein persistieren können, sondern insbesondere aus den sekundären Auswirkungen auf die Entwicklung der Persönlichkeit und auf die Chancen in Schule und Beruf. Einschränkungen der sozialen Prognose sind Folge der Beeinträchtigung des Schulerfolgs. Viele LRS-Kinder verlassen vorzeitig die Schule und selbst bei guter Intelligenz (IQ >112) legen nur 10\% das Abitur ab (Strehlow 2004). Der Schulerfolg der LRS-Kinder entspricht somit nicht ihren allgemeinen intellektuellen Fähigkeiten, sondern ist vergleichbar mit dem von minderbegabten Regelschülern (Esser 1991). Als Folge fehlender oder niedriger Schulabschlüsse schließen nur 50 \% der LRS-Kinder eine Berufs- und lediglich $1 \%$ eine akademische Ausbildung ab (Maughan 1995).

Die ICD-1o definiert umschriebene Entwicklungsstörungen der motorischen Funktionen (F82) als eine schwerwiegende Beeinträchtigung der Entwicklung der motorischen Koordinationsfähigkeit, die nicht allein durch eine Intelligenzminderung oder eine angeborene oder erworbene neurologische Störung erklärbar und nicht direkte Auswirkung von Seh- oder Hörstörungen ist. Die Störung 
ist nach der ICD-10 dadurch charakterisiert, dass die motorische Koordinationsfähigkeit des Kindes bei fein- oder grobmotorischen Anforderungen deutlich unterhalb des Niveaus liegt, das nach dem Alter und der allgemeinen Intelligenz zu erwarten wäre. Das Muster der motorischen Beeinträchtigung variiert und ist altersabhängig. Die Kinder wirken insgesamt unbeholfen und erwerben verzögert Laufen, Hüpfen und Schwimmen. Sie erlernen nur mühsam Schuhe binden, Treppen steigen, Dreirad oder Fahrrad fahren und sind ungeschickt beim Werfen und Fangen von Bällen. Die Kinder stolpern und fallen oft, zeichnen ungeschickt und lassen Sachen häufig zu Boden fallen. Ihr Schriftbild ist krakelig und schlecht leserlich.

Psychische Auffälligkeiten und eine Komorbidität mit ADHS sind vorwiegend bei Kindern mit Sprachentwicklungsstörungen und/oder Lese-Rechtschreibstörungen genauer untersucht worden, weshalb sich die folgenden Ausführungen auf diese beiden Störungsbilder konzentrieren.

\subsection{Psychische Auffälligkeiten bei umschriebenen Entwicklungsstörungen}

\section{Häufigkeit und Art}

Psychische Auffälligkeiten wurden bei sprachentwicklungsgestörten Kindern vielfach beschrieben. Die Häufigkeit wird mit 30 bis $50 \%$ angegeben. In einer eigenen Studie beurteilten die Mütter von 209 sprachauffälligen Vorschulkindern das Verhalten ihrer Kinder mit dem Verhaltensfragebogen (E-F) von Meyer-Probst (1983). Bei 44\% der Kinder lag der Gesamtwert im auffälligen Bereich (s. Abb. 1). ADHS und soziale Anpassungsstörungen standen im Vordergrund. Häufig wurde zusätzlich über emotionale Störungen berichtet (s. Abb. 2).

Bei Jugendlichen und Erwachsenen mit Sprachentwicklungsstörungen im Kindesalter wurden Schwächen beim Erkennen von Emotionen und beim Lösen sozialer Probleme beschrieben (Bishop 1997; Cohen \& Horodezky 1998). Insgesamt ist das Risiko psychiatrischer Auffälligkeiten bei sprachentwicklungsgestörten Kindern etwa 4- bis 5-mal so groß wie bei Kindern ohne Entwicklungsbesonderheiten (Beitchman et al. 1990; Noterdaeme \& Amorosa 1998). Eine Beziehung zwischen der Schwere der Sprachstörung und dem Ausprägungsgrad der Verhaltensauffälligkeiten wurde nicht beobachtet (Benasich et al. 1993; v. Suchodoletz \& Keiner 1998).

Eine Verbindung zwischen Sprachentwicklungsstörung und späterem dissozialem Verhalten legen Untersuchungen bei obdachlosen Jugendlichen, bei Jugendlichen mit Schuleschwänzen, bei Gefängnisinsassen und bei vorbestraften Frauen nahe, bei denen eine Häufung von Sprachentwicklungsstörungen in der Anamnese gefunden wurde. Allerdings scheinen diese nicht unmittelbar ein Risiko für dissoziales Verhalten im Jugend- und jungen Erwachsenenalter darzustellen. Eine antisoziale Fehlentwicklung wurde nur bei 
einer Kombination mit psychosozialen Risiken (niedriger sozioökonomischer Status, sehr junge Mutter, mütterliche Depression u.a.) beobachtet (Naylor et al. 1994).

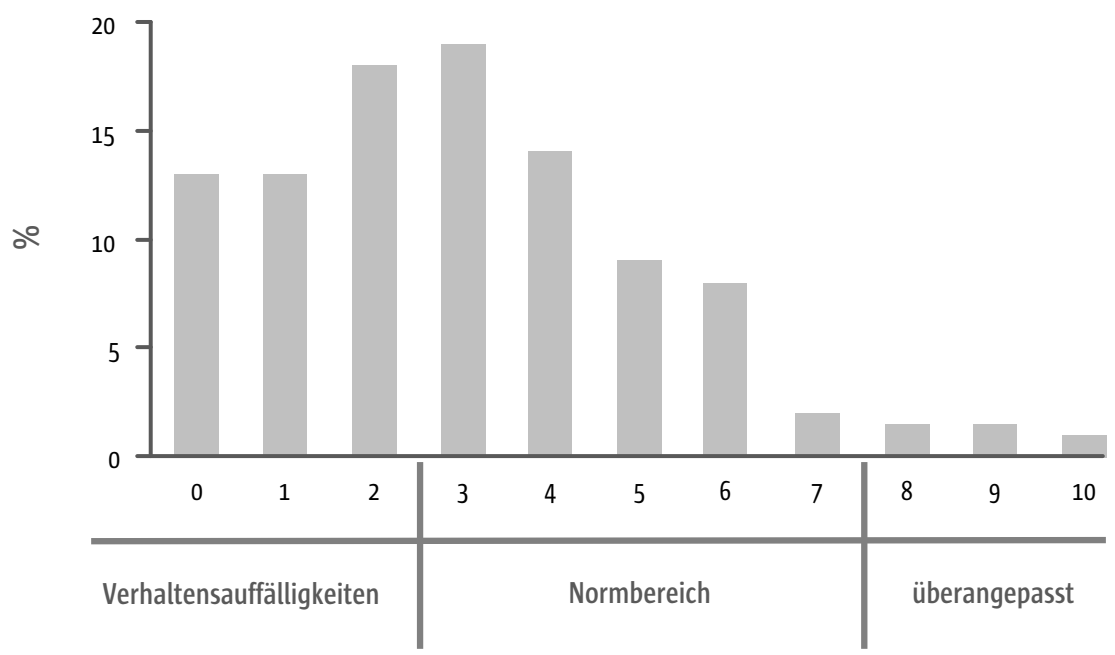

C-Wert Gesamtscore

Abb. 1 Häufigkeit von Verhaltensauffälligkeiten bei sprachgestörten Vorschulkindern ( $n=209)$ nach Einschätzung der Mütter (Verhaltensfragebogen E-F von Meyer-Probst)

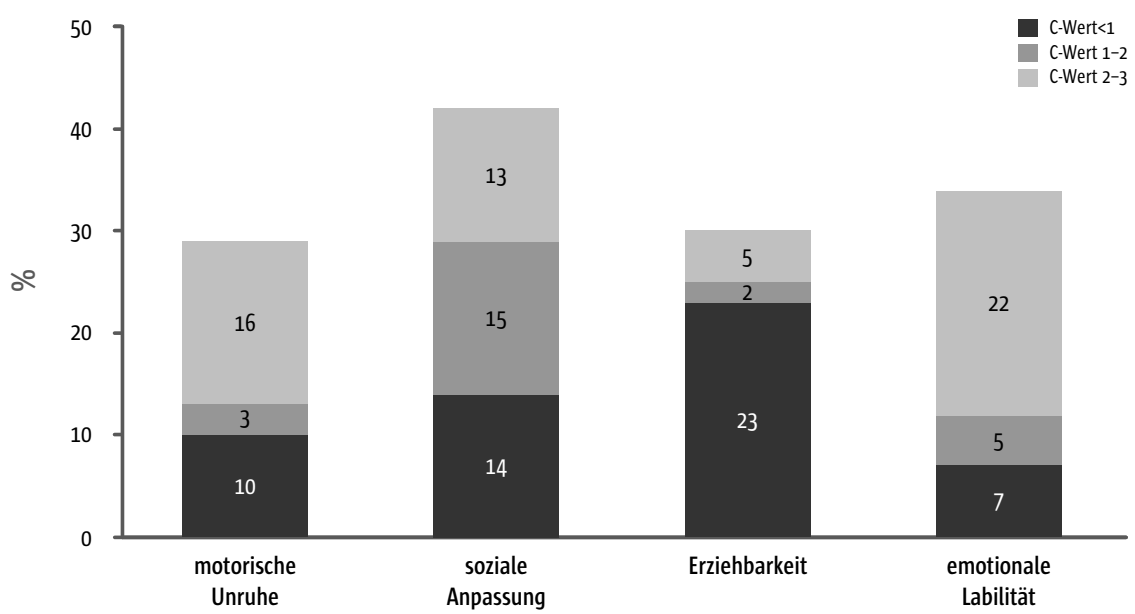

Abb. 2 Häufigkeit einzelner Verhaltensauffälligkeiten bei sprachgestörten Vorschulkindern ( $n=209$ ) nach Einschätzung der Mütter (Verhaltensfragebogen E-F von Meyer-Probst)

Die Prognose hinsichtlich der langfristigen psychischen Entwicklung war bei Jungen deutlich schlechter als bei Mädchen. Im frühen Erwachsenenalter wurde bei Männern antisoziales Verhalten 10-mal häufiger als bei Frauen re- 
gistriert, während Angststörungen bei beiden Geschlechtern etwa gleich häufig festzustellen waren ( 26 bzw. $28 \%$ ). Im Gegensatz zu antisozialem Verhalten wurde keine signifikante Erhöhung von Substanzmissbrauch beobachtet. Jugendliche Abhängige mit einer Sprachentwicklungsstörung zeigten jedoch eine höhere Komorbidität mit psychiatrischen Auffälligkeiten (insbesondere antisoziale Persönlichkeits- und internalisierende Störungen) und einen geringeren Grad an sozialer Anpassung als solche ohne Sprachprobleme (Beitchman et al. 1999).

Besonders ungünstige Verläufe hinsichtlich des Kommunikations- und Sozialverhaltens können bei Kindern mit ungewöhnlich ausgeprägten Sprachentwicklungsstörungen auftreten. Mawhood et al. (2000) beschrieben bei einigen dieser Kinder im jungen Erwachsenenalter ein autismusähnliches Verhalten. Auch noch im mittleren Erwachsenenalter waren sie hinsichtlich sozialer Kompetenz und Einfühlungsvermögen sowohl den Probanden der Kontrollgruppe als auch ihren Geschwisterkindern unterlegen. Auch über das Auftreten von schizo-affektiven Erkrankungen im Erwachsenenalter wurde berichtet.

Sprachstörungen scheinen aber nicht auf direktem Weg zu psychischen Fehlentwicklungen zu führen. Pfadmodelle auf der Crundlage der Ergebnisse einer groß angelegten neuseeländischen Longitudinalstudie, in welcher die Entwicklung der Kinder bis zum 18. Lebensjahr verfolgt wurde, sprechen dafür, dass die Wirkungen von Sprachstörungen auf das Verhalten über LeseRechtschreibprobleme, geringe verbale Selbstregulation, Aufmerksamkeitsstörung und Schulversagen gefolgt von niedrigem Ausbildungsstand vermittelt werden. Nicht die Sprachstörung selbst, sondern die Lesefähigkeit zu Schulbeginn erwies sich als Prädiktor für spätere Verhaltensbesonderheiten und umgekehrt beeinflusste antisoziales Verhalten die spätere Lesefähigkeit (Fergusson \& Lynskey 1997).

Dass psychische Auffälligkeiten, insbesondere eine ADHS-Symptomatik, bei LRS-Kindern häufiger als bei Kindern ohne Schulprobleme anzutreffen sind, ist seit Langem bekannt (Hinshaw 1992). Vielen LRS-Kindern fällt es schwer, sich auf Aufgaben zu konzentrieren und sie sind durch Störreize leicht ablenkbar. Sie sind zappelig, erscheinen innerlich unausgeglichen und das Stillsitzen fällt ihnen schwer. Manche Kinder neigen zu verstärkten Trotzreaktionen, Wutanfällen und aggressivem Verhalten. Eine LRS wird auch als Risiko für delinquente Fehlentwicklungen angesehen. Immer wieder beschrieben wurden Leistungsängste, überempfindliche Reaktionen bei Kritik und eine Neigung zu Verstimmungszuständen. Viele LRS-Kinder entwickeln Schulunlust und Schulangst. Bei Leistungsanforderungen zeigen sie wenig Selbstvertrauen. „Das kann ich nicht“ ist eine häufige Antwort und bei ersten Schwierigkeiten resignieren sie oder bitten um Unterstützung. Im Zusammenhang mit schulischen Anforderungen wird über Übelkeit, Bauchschmerzen oder Erbrechen geklagt. Die Kinder geben Kopfschmerzen an und neigen zu Schlafstörungen. Ein sekundäres Einnässen kann auftreten. Die Vielfältigkeit psychischer Auffälligkeiten bei LRS-Kin- 
dern wird aus einer Studie von Goldston et al., (2007) deutlich. Diese Arbeitsgruppe verglich die Häufigkeit psychiatrischer Störungen bei 15-jährigen lese-rechtschreibgestörten Jugendlichen mit einer gleichaltrigen Kontrollgruppe (s. Abb. 3).

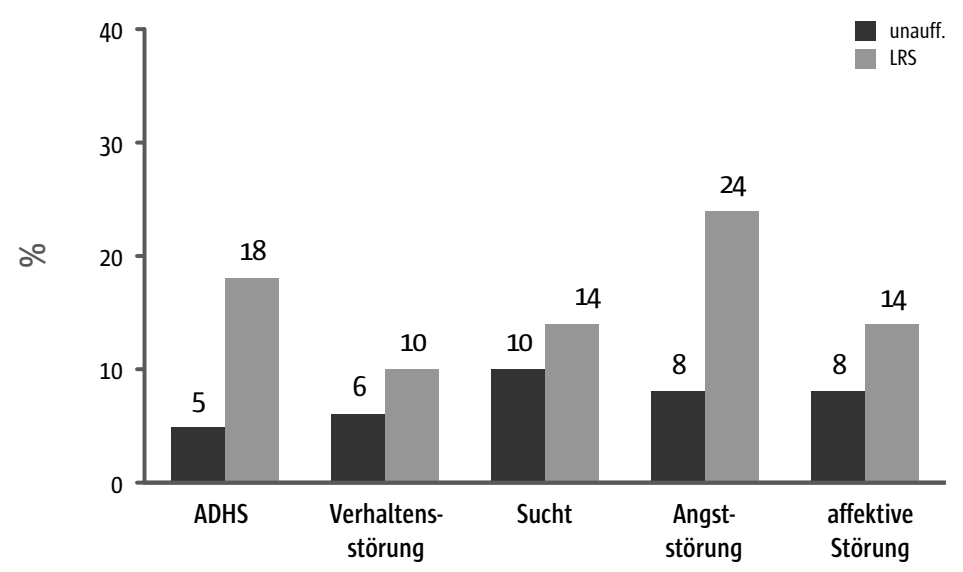

Abb. 3 Häufigkeit psychiatrischer Störungen bei 15-jährigen lese-rechtschreibgestörten Jugendlichen im Vergleich zu einer unauffälligen Kontrollgruppe ( $n=94$ pro Gruppe) nach Goldston et al. (2007)

Eine Häufung eines ADHS wird bei LRS-Kindern immer wieder beschrieben und umgekehrt wird bei Kindern mit einem hyperkinetischen Syndrom gehäuft eine LRS festgestellt. Störungen des Sozialverhaltens bis hin zu dissozialen Verhaltensweisen (Lügen, Stehlen, aggressives Verhalten) werden bei etwa $25 \%$ der Jugendlichen und jungen Erwachsenen mit einer LRS angegeben (Esser 1991). Leseprobleme im Alter von 9 Jahren erwiesen sich als Prädiktor für spätere Verhaltensstörungen (Williams \& McGee 1994). Von einigen Autoren wird die Legasthenie als wesentliche Ursache einer kriminellen Entwicklung angesehen. Ob Probleme beim Schriftspracherwerb aber tatsächlich zu delinquentem Verhalten führen, ist umstritten. Cornwall \& Bawden (1992) kamen aufgrund einer Übersicht der relevanten Literatur zu dem Ergebnis, dass dissoziales Verhalten schlechte Schulleistungen - insbesondere bei sprachlichen Anforderungen - zur Folge hat, aber nicht umgekehrt. Schlechte Leser mit schwacher Intelligenz zeigen im frühen Schulalter vermehrt aggressives Verhalten und Delinquenz in der Pubertät sowie schlechtere Schulleistungen als vom IQ zu erwarten wären. Hingegen wurde bei schlechten Lesern mit guter Intelligenz, also Kindern mit einer umschriebenen LRS, eine Häufung antisozialen Verhaltens nicht belegt.

Über die Häufigkeit abnormer Ängste und depressiver Verstimmungen (emotionale Störungen) liegen widersprüchliche Angaben vor. In klinischen Stichproben wurden bei Kindern mit einer LRS gehäuft depressive Störungen beschrieben (Willcutt \& Pennington 200o), während in epidemiologischen Studien vermehrt Angststörungen, nicht aber depressive Störungen beobachtet 
wurden (Carroll et al. 2005). Unter den Angststörungen stehen soziale Ängste ganz im Vordergrund (Goldston et al. 2007). In der Kurpfalzstudie wurden bei LRS-Kindern im frühen Schulalter verstärkt Ängste und psychosomatische Symptome beobachtet (Esser 1991). Von anderen Autoren wurden im späteren Schulalter ein niedriges Selbstwertgefühl und eine Erhöhung des allgemeinen Angstpegels beschrieben. Eine erhöhte Selbstmordrate wird immer wieder erwähnt, ist aber nicht belegt. Diese psychischen Störungen sind aber nicht LRS-spezifisch. Ähnliche Entwicklungen in Richtung einer emotionalen Verunsicherung werden auch bei Jugendlichen mit allgemeinen Lernstörungen beobachtet.

\section{Ursachen psychischer Auffälligkeiten}

Um die Frage zu klären, ob emotionale und Verhaltensbesonderheiten bei Kindern mit umschriebenen Entwicklungsstörungen primär bestehen oder ob sie sich erst als Folge der Kommunikations- bzw. Schulprobleme herausbilden, wurde die psychische Entwicklung betroffener Kinder über den Verlauf mehrerer Jahre beobachtet. In diesen Längsschnittstudien wurde davon ausgegangen, dass von der Entwicklungsstörung unabhängig auftretende psychische Störungen (Komorbidität) über die Jahre weitgehend stabil bleiben, während psychoreaktiv bedingte psychische Auffälligkeiten (sekundäre Neurotisierung) eine deutliche Zunahme zeigen.

Die Arbeitsgruppe um Beitchman (2001) verfolgte die psychische Entwicklung sprach- und sprechgestörter Kinder vom 5. bis zum 19. Lebensjahr. Das Verhalten von 77 Kindern mit Sprachentwicklungs- und 38 mit umschriebenen Artikulationsstörungen wurde im Verlauf mit dem von 129 unauffällig entwickelten Kindern verglichen. Bei den sprachentwicklungsgestörten Kindern zeigte sich eine kontinuierliche Zunahme psychiatrischer Auffälligkeiten. Im jungen Erwachsenenalter lag bei ihnen die Rate psychischer Störungen mit $40 \%$ doppelt so hoch wie in der Kontrollgruppe. Insbesondere Angststörungen (27\% gegenüber $8 \%$ ) und antisoziale Persönlichkeitsstörungen (20\% gegenüber $8 \%$ ) wurden deutlich häufiger angetroffen. Kinder mit umschriebenen Artikulationsstörungen hingegen zeigten keine Erhöhung der Anzahl psychiatrischer Diagnosen. Wenn psychische Auffälligkeiten bestanden, dann traten sie in der Regel in Kombination auf. Die Art der Komorbidität unterschied sich zwischen den Gruppen nicht.

Hinsichtlich der Angststörungen wurde in allen drei Gruppen in ähnlicher Weise eine Zunahme bis zum 8. Lebensjahr beobachtet. Danach blieb die Häufigkeit in der Kontrollgruppe auf etwa dem gleichen Niveau bestehen, während bei den Kindern mit Sprech- und Sprachstörungen ein weiterer Anstieg eintrat. Bei Kindern mit umschriebenen Artikulationsstörungen wurde etwa mit dem 11. Lebensjahr ein Plateau erreicht, während in der Gruppe der sprachentwicklungsgestörten Kinder eine kontinuierliche Zunahme bis zum Ende des Beobachtungszeitraumes mit 19 Jahren nachzuweisen war. Unter den Angststörungen traten vorrangig soziale Phobien auf. Aber auch Platzangst 
und andere einfache Phobien waren in der Gruppe der Sprachgestörten vermehrt anzutreffen.

Vergleichbare Entwicklungen wurden bei Kindern mit einer Lese-Rechtschreibstörung beschrieben. In einer Studie von Gasteiger-Klicpera et al. (2006) wurde das Verhalten von Kindern mit und ohne LRS vom Ende der Kindergartenzeit bis zum Ende der 4. Klasse beobachtet. Die Verhaltenseinschätzung erfolgte einerseits durch Kindergärtnerinnen bzw. Lehrerinnen und andererseits durch andere Kinder der Gruppe. Die Ergebnisse demonstrieren sehr klar, dass hyperkinetisches und aggressives Verhalten bei Kindern mit einer LRS bereits im Kindergartenalter vermehrt zu beobachten ist, im Laufe der Jahre aber kaum zunimmt. Im Gegensatz dazu unterscheiden sich lese-rechtschreibgestörte und andere Kinder hinsichtlich Sozialkontakt und prosozialem Verhalten anfangs kaum. Ende der 4. Klasse hingegen fallen die LRS-Kinder durch eine vermehrte Zurückgezogenheit und ein geringeres prosoziales Verhalten auf.

Die bisherigen Erfahrungen sprechen somit dafür, dass bei Kindern mit umschriebenen Entwicklungsstörungen hyperkinetisches und oppositionelles Verhalten eher als zusätzliche, von der Entwicklungsstörung unabhängige Auffälligkeiten (Komorbidität) aufzufassen sind, während sich soziale Ängste erst als Folge der Entwicklungsstörung im Laufe der Zeit entwickeln.

Als Ursache für die häufige Komorbidität von LRS und ADHS werden genetische Faktoren angesehen. Zwillingsstudien sprechen dafür, dass bei einem gleichzeitigen Vorkommen von Entwicklungsstörung und ADHS eine genetische Disposition sowohl für die Entwicklungs- als auch die hyperkinetische Störung vorliegt.

Hintergründe der Zunahme internalisierender Störungen und insbesondere sozialer Ängste sind zum einen immer wiederkehrende Misserfolgserlebnisse und zum anderen Stigmatisierungsprozesse, die in den letzten Jahren zunehmende Aufmerksamkeit gefunden haben. Aus der soziologischen Literatur ist bekannt, dass für das Ausmaß von Stigmatisierung Sichtbarkeit einer Normabweichung und der Grad der Beeinträchtigung der Kommunikationsfähigkeit von entscheidender Bedeutung sind. Ist der Verlauf des Interaktionsprozesses schwer vorhersehbar, so führt dies zu einer Verunsicherung der Partner, wodurch Ablehnung und Ausgrenzung provoziert werden. Knox und Conti-Ramsden (2003) gingen der Frage nach, welchen sozialen Rang sprachgestörte Kinder in der Gruppe Gleichaltriger einnehmen. Mittels Fragebogen erfassten sie bei 100 sprachentwicklungsgestörten 11-jährigen Kindern, ob diese sich in der Gruppe als „Prügelknabe“ erleben. Die sprachgestörten Kinder gaben gegenüber denen der Kontrollgruppe 3-mal so häufig ( $36 \mathrm{zu}$ 12\%) an, dass sie von ihren Klassenkameraden terrorisiert und schikaniert werden. Ähnlich wird bei lese-rechtschreibgestörten Kindern sowohl nach dem Eindruck der Lehrerinnen als auch nach dem Erleben der Klassenkameraden im Laufe der Grundschulzeit eine zunehmende Viktimisierung dieser Kinder beobachtet (Gasteiger-Klicpera et al. 2006).

Wie häufig Stigmatisierung von Eltern sprachgestörter Kinder in den Bereichen Kindergruppen, Familie und weiterem Umfeld wahrgenommen wird, 
untersuchten wir mittels Elternfragebögen. Von 386 Eltern gaben etwa $50 \%$ an, negative Reaktionen des Umfeldes gegenüber ihrem Kind aufgrund der Entwicklungsauffälligkeiten zu beobachten. Über herabsetzendes Verhalten und Ausgrenzung wurde am häufigsten in Kindergruppen, aber auch in der eigenen Familie berichtet. Auch die Eltern selbst fühlten sich häufig in den Stigmatisierungsprozess einbezogen (Suchodoletz \& Machery 2006). Als ein wesentlicher, das Ausmaß von Stigmatisierung beeinflussender Faktor hat sich in unserer Untersuchung das Verhalten der Kinder erwiesen. Der Score für auffälliges Sozialverhalten korrelierte hoch signifikant sowohl mit dem Stigma-Score der Kinder als auch mit dem der Eltern. Aus der Bullying-Forschung ist bekannt, dass Kinder mit einem ADHS, mit Störungen des Sozialverhaltens oder einem Asperger Syndrom nicht nur häufig andere Kinder drangsalieren, sondern auch ein erhöhtes Risiko tragen, selbst Opfer von Bullying zu werden (Spitczok von Brisinski 2005). Wie negativ sich insbesondere Verhaltensabweichungen im sozialen Kontext auswirken, geht auch daraus hervor, dass in der Schule der soziale Status von Kindern mit Verhaltensstörungen noch niedriger ist als der von Kindern mit körperlichen oder Sinnesbeeinträchtigungen (Dumke et al. 1993).

Aber nicht nur selbst wahrgenommene Verhaltensabweichungen, sondern schon alleine deren Erwartung kann Anlass zu Diskriminierung sein. Milich et al. (1992) beobachteten den Interaktionsprozess zwischen Paaren von Kindern, die einander nicht kannten. Einigen Kindern wurde gesagt, dass ihr Interaktionspartner Verhaltensprobleme hätte. In diesen Fällen wurde das als verhaltensgestört bezeichnete Kind weniger freundlich behandelt und seltener ins Spiel einbezogen als Kinder, deren Interaktionspartner diese Information nicht erhalten hatten. Die betroffenen Kinder spürten die Ablehnung und verstärkten wiederum durch ihr eigenes Verhalten die negativen Reaktionen.

Sozialer Rückzug und das Gefühl, abgelehnt zu werden, kann aber auch Folge von Selbststigmatisierung sein. Betroffene befürchten negative Reaktionen, werten Äußerungen anderer übermisstrauisch und vermeiden deshalb den Umgang mit denjenigen, von denen sie Ausgrenzung erwarten. Allerdings korreliert die Erwartung von Ablehnung nicht unbedingt mit den tatsächlichen Einstellungen des Umfeldes. In einer Studie von Norvilitis et al. (2002) zeigte sich, dass Mütter von Kindern mit einem ADHS davon ausgehen, dass andere Mütter negativ gegenüber hyperkinetischen Kindern eingestellt sind. Eine Befragung von Müttern unauffällig entwickelter Kinder ergab jedoch, dass dies häufig gar nicht der Fall ist und somit Befürchtungen betroffener Eltern nicht immer begründet sind.

Die Häufigkeit der Einbeziehung von Eltern entwicklungsauffälliger Kinder in den Stigmatisierungsprozess erklärt sich insbesondere daraus, dass Eltern nicht selten für Entwicklungsverzögerungen oder Verhaltensauffälligkeiten ihrer Kinder als verantwortlich angesehen werden. Ihnen wird ein unzureichendes familiäres Engagement und Inkompetenz bei der Erziehung vorgeworfen. Wie in der soziologischen Forschung gezeigt werden konnte, werden 
unter auffälligen Personen insbesondere diejenigen von anderen abgelehnt und ausgegrenzt, denen eine eigene Verantwortung für die Störung zugeschrieben wird (Cloerkes 200o). Crandall u. Moriarty (1995) untersuchten das Ausmaß sozialer Ablehnung bei Patienten mit unterschiedlichen Krankheitsbildern. Als entscheidender Faktor erwies sich neben der Schwere des Störungsbildes der Grad der angenommenen eigenen Verantwortlichkeit für die Erkrankung.

Eine Entwicklungsverzögerung stellt nicht nur für das betroffene Kind, sondern auch auf das Umfeld einen Belastungsfaktor dar. Wie eine Befragung von Müttern von 138 sprachgestörten Kindern ergeben hat, sehen diese nicht nur bei sich selbst, sondern auch bei anderen Familienangehörigen deutliche Belastungsreaktionen als Folge der Entwicklungsstörung eines ihrer Kinder (s. Abb. 4). Die Mütter machen sich Sorgen um die Zukunft ihres Kindes und viele empfinden Niedergeschlagenheit und Enttäuschung, gelegentlich aber auch aggressive Gefühle aufgrund der Entwicklungsbesonderheiten ihres Kindes (s. Abb. 5). Derartige im Rahmen eines Bewältigungsprozesses üblichen Ängste und emotionalen Reaktionen sollten bei Beratungsgesprächen unbedingt thematisiert werden (Limm \& Suchodoletz 1998).
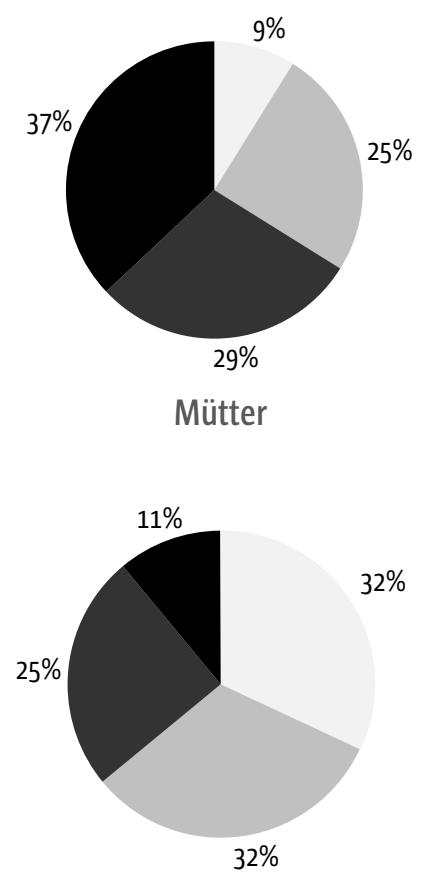

Geschwister

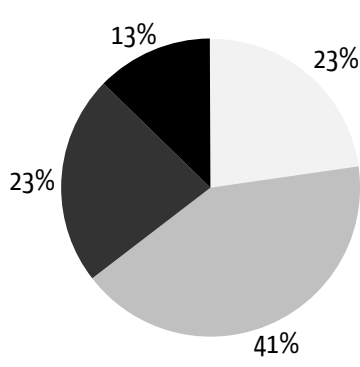

Väter

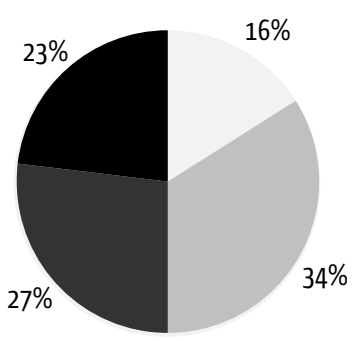

Kind selbst

Abb. 4 Belastung einzelner Familienmitglieder durch eine Sprachentwicklungsstörung des Kindes (Angaben der Mütter, $n=138$ ) 

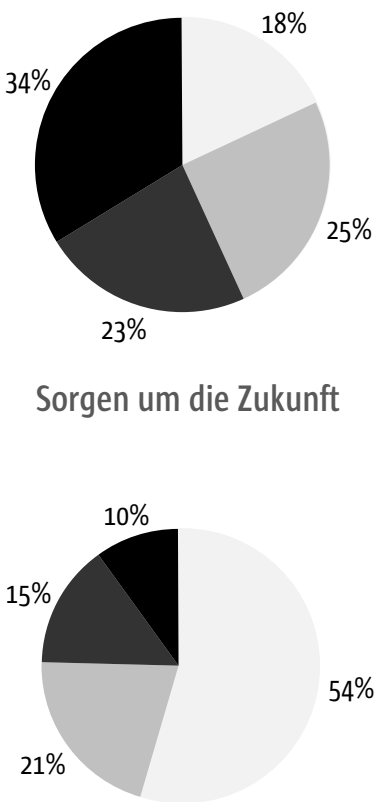

Enttäuschung

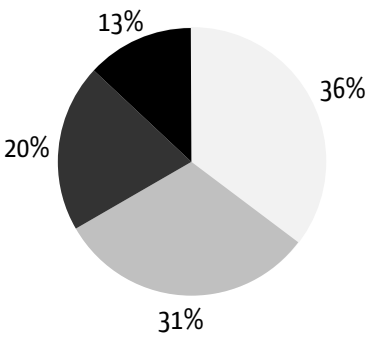

Niedergeschlagenheit

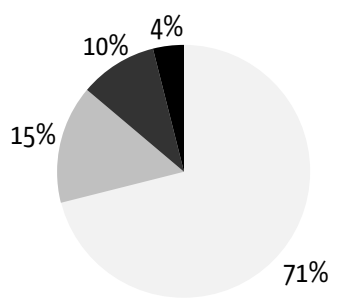

aggressive Gefühle

Abb. 5 Ängste und Empfindungen von Müttern sprachentwicklungsgestörter Kinder ( $n=138)$

\subsection{Medikamentöse Therapie}

Vielfache Versuche wurden unternommen, die Lernfähigkeit von Kindern mit Entwicklungsstörungen, insbesondere von Kindern mit einer LRS, zu verbessern. Der Verordnung von Medikamenten liegt der Gedanke zugrunde, dass es sich bei Entwicklungsstörungen um eine hirnorganisch bedingte Leistungsminderung handelt. Die Medikation soll zu einer allgemeinen Verbesserung der geistigen Fähigkeiten und damit verbunden der Lernfähigkeit beim Lautund Schriftspracherwerb führen. Bei LRS-Kindern wurden bislang insbesondere Psychostimulanzien, Nootropika und Tranquilizer auf ihre Wirksamkeit hin überprüft.

Die Wirkung von Methylphenidat, als Hauptvertreter der Gruppe der Psychostimulanzien, wurde in den 1980er Jahren mehrfach bei LRS-Kindern überprüft. Es konnten nur geringe bzw. keine relevanten Verbesserungen der Leseleistungen von LRS-Kindern nachgewiesen werden (Gittelman et al. 1983; Aman \& Werry 1982). Die Lese-Rechtschreibstörung zählt deshalb auch nicht zu den Indikationen von Psychostimulanzien (AACAP 2002). Im Gegensatz zu Kindern mit einer reinen Lese-Rechtschreibstörung ist beim Vorliegen eines hyperkinetischen Syndroms die Behandlung mit Methylphenidat äußerst effektiv. In über 16o Doppelblindstudien mit insgesamt etwa 5000 Kindern konnte nachgewie- 
sen werden, dass sich Methylphenidat bei 70-80\% der hyperkinetischen Kinder positiv auf die Lernfähigkeit und das Verhalten auswirkt. Da eine hyperkinetische Störung bei vielen Kindern mit Entwicklungsstörungen als zusätzliche Symptomatik nachweisbar ist, kann die Verordnung von Psychostimulanzien auch bei entwicklungsgestörten Kindern sinnvoll sein (Dykman \& Ackerman 1991). In einer Übersichtsarbeit kommen Beitchman \& Young (1997) zu dem Ergebnis, dass Methylphenidat bei LRS-Kindern mit einem Aufmerksamkeitsmangelsyndrom die Lesefähigkeit verbessert und die Kinder in die Lage versetzt, im Unterricht konzentrierter mitzuarbeiten und Aufgaben zu beenden.

Zu den Nootropika werden Medikamente gerechnet, die durch eine Verbesserung des Hirnstoffwechsels positive Auswirkungen auf Gedächtnis, Konzentrations- und Lernfähigkeit ausüben sollen. Sie werden insbesondere bei chronischen hirnorganischen Erkrankungen und in der Rehabilitationsphase nach Schädel-Hirn-Traumen, Schlaganfall und anderen hirnorganisch bedingten Leistungsminderungen eingesetzt. Unter der Vorstellung, dass Entwicklungsstörungen hirnorganisch erklärbare Lernschwächen darstellen, kommen Nootropika auch bei Entwicklungsstörungen zum Einsatz. Das am häufigsten verwendete Nootropikum ist Piracetam.

In den 1980er Jahren wurde der Frage der Wirksamkeit von Nootropika bei der Behandlung einer Lese-Rechtschreibstörung in mehreren Studien nachgegangen. Im deutschsprachigen Raum wurde meines Wissens in jüngster Zeit nur eine Arbeit publiziert, die zu dem Ergebnis kam, dass sich Lese- und Rechtschreibleistungen sowie die Fähigkeit zur sprachlichen Informationsverarbeitung unter Piracetam verbessern (Häßler \& Tilch 1996). Da diese Studie aber offen angelegt war und eine Kontrollgruppe fehlte, ist deren Aussagefähigkeit begrenzt. Doppelblindstudien aus dem angloamerikanischen Raum zeigten widersprüchliche Resultate. Insgesamt sprechen die Ergebnisse dafür, dass Nootropika zu einer Erhöhung der Lesegeschwindigkeit, nicht aber zu einer Verbesserung von Leseverständnis oder einer Verminderung von Leseund Rechtschreibfehlern führen. Eine Übersicht über kontrollierte PiracetamStudien mit Doppelblinddesign, die insgesamt etwa 750 Kinder einschlossen, stellten Wilsher-Colin \& Taylor (1994) zusammen. Da unter Piracetam relevante Nebenwirkungen nicht zu befürchten sind, kommen die Autoren trotz der geringen positiven Effekte auf die Lesefähigkeit zu dem Schluss, dass ein Einsatz des Medikaments bei Kindern mit einer LRS gerechtfertigt sei.

Die Hauptindikationen für Tranquilizer sind akute und chronische Angstzustände. Behandlungsversuche mit Tranquilizern gehen von der Annahme aus, dass die Lese-Rechtschreibstörung Ausdruck einer Lernblockade infolge von Versagensangst beim Lesen und Schreiben sei. Unter den Tranquilizern ist Diazepam das Standardpräparat. Tranquilizer erwiesen sich aber nach den wenigen vorliegenden Erfahrungen bei Kindern mit einer Lese-Rechtschreibstörung als unwirksam (Aman \& Werry 1982).

Zusammenfassend lässt sich sagen, dass in mehreren gut kontrollierten Studien nachgewiesen werden konnte, dass sich die Lese-Geschwindigkeit bei LRS-Kindern durch Medikamente verbessern lässt. Lese- und Rechtschreib- 
fehler und das Leseverständnis hingegen sind medikamentös bislang kaum zu beeinflussen. Da die Effekte aber insgesamt gering sind, ist die Verordnung von Medikamenten bei einer isolierten Lese-Rechtschreibstörung nicht angebracht (Warnke et al. 2007). Wenn jedoch bei einem LRS-Kind gleichzeitig ein ADHS besteht, wie dies relativ häufig der Fall ist, dann kann eine Einnahme von Methylphenidat durchaus hilfreich sein. Hyperkinetische Kinder werden unter Psychostimulanzien meist wesentlich konzentrierter, lernfähiger und weniger verhaltensauffällig, so dass erst durch die Pharmakotherapie die Voraussetzungen für Lernfortschritte geschaffen werden. Wie durch kontrollierte Studien gezeigt wurde, sprechen LRS-Kinder mit einem ADHS genauso gut wie andere hyperkinetische Kinder auf eine Methylphenidat-Gabe an.

Hinsichtlich einer Behandlung mit Nootropika (z. B. Piracetam) liegen mehrere kontrollierte Studien vor. Diese belegen, dass unter Nootropika eine Erhöhung der Lesegeschwindigkeit eintritt, während sich Lesefehler, Leseverständnis und Rechtschreibfähigkeit kaum verändern. Die Wirkung auf die Lesegeschwindigkeit ist am ehesten auf das aktivierende Potenzial von Medikamenten dieser Gruppe zurückzuführen. Eine echte Steigerung der Lernfähigkeit im Schriftsprachbereich ist jedoch nicht zu erreichen. Die Wirksamkeit von Nootropika ist allerdings auch bei anderen Hirnerkrankungen eher gering bzw. zweifelhaft. Eine kritische Übersicht zu Arzneimittelwirkungen kommt deshalb zu dem Fazit: „Für Nootropika gibt es keine Indikation“ (transparenz-telegramm 1990/91).

Über die Wirksamkeit von Benzodiazepinen und anderen Medikamenten in der LRS-Therapie liegen nur wenige Mitteilungen vor, die keine Hinweise darauf liefern, dass sich die Lese- und Rechtschreibfähigkeit nennenswert verbessern lässt.

Eine effektive medikamentöse Behandlung von umschriebenen Entwicklungsstörungen ist somit bislang nicht bekannt. Eine medikamentöse Therapie entwicklungsauffälliger Kinder ist nur dann indiziert, wenn dies aufgrund zusätzlicher psychischer Störungen erforderlich wird.

\section{Literatur}

Aman MG, Werry IS. Methylphenidate and diazepam in severe reading retardation. Journal of the American Academy of Child and Adolescent Psychiatry 1982;21(1):31-7.

American Academy of Child and Adolescent Psychiatry. Practice parameters for the use of stimulant medications in the treatment of children, adolescents, and adults. Journal of the American Academy of Child and Adolescent Psychiatry 2002;41Suppl.:26S-49S.

Beitchman JH, Douglas L, Wilson B, Johnson C, Young A, Atkinson L, et al. Adolescent substance use disorders: findings from a 14-year follow-up of speech/language-impaired and control children. Journal of Clinical Child Psychology 1999;28(3):312-21.

Beitchman JH, Hood J, Inglis A. Psychiatric risk in children with speech and language disorders. Journal of Abnormal Child Psychology 1990;18:283-296.

Beitchman JH, Wilson B, Johnson Cl, Atkinson L, Young A, Adlaf E, et al. Fourteen-year follow-up of speech/ language-impaired and control children: Psychiatric outcome. Journal of the American Academy of Child and Adolescent Psychiatry 2001;40:75-82. 
Beitchman JH, Young AR. Learning disorders with a special emphasis on reading disorders: A review of the past 10 years. Journal of the American Academy of Child and Adolescent Psychiatry 1997;36:1020-1032.

Benasich AA, Curtiss S, Tallal P. Language, learning, and behavioral disturbances in childhood: A longitudinal perspective. Journal of the American Academy of Child and Adolescent Psychiatry 1993;32:585-594.

Bishop DVM. Uncommon understanding - Development and disorders of language comprehension in children. Hove: Psychology Press; 1997.

Carroll JM, Maughan B, Goodman R, Meltzer H. Literacy difficulties and psychiatric disorders: evidence for comorbidity. Journal of Child Psychology and Psychiatry 2005;46(5):524-32.

Cloerkes G. Die Stigma-Identitäts-These. Gemeinsam leben - Zeitschrift für integrative Erziehung 2000;3:104-111.

Cohen N), Horodezky NB. Language impairments and psychopathology. Journal of the American Academy of Child and Adolescent Psychiatry 1998;37:46-462.

Cornwall A, Bawden HN. Reading disabilities and aggression: a critical review. Journal of Learning Disabilities 1992;25(5):281-8.

Crandall CS, Moriarty D. Physical illness stigma and social rejection. British Journal of Social Psychology 995;34:67-83.

Dumke D, Schaefer G, Osinski M, Wirtz E. Entwicklung behinderter und nichtbehinderter Schüler in Integrationsklassen. Einstellungen, soziale Beziehungen, Persönlichkeitsmerkmale und Schulleistungen. Weilheim: Deutscher Studien Verlag; 1993.

Dykman RA, Ackerman PT. Attention deficit disorder and specific reading disability: Separate but often overlapping disorders. Journal of Learning Disabilities 1991;24:96-103.

Esser G. Was wird aus Kindern mit Teilleistungsschwächen? Der langfristige Verlauf umschriebener Entwicklungsstörungen. Stuttgart: Ferdinand Enke Verlag; 1991.

Fergusson DM, Lynskey MT. Early reading difficulties and later conduct problems. Journal of Child Psychology and Psychiatry 1997;38(8):899-907.

Gasteiger Klicpera B, Klicpera C, Schabmann A. Der Zusammenhang zwischen Lese-, Rechtschreib- und Verhaltensschwierigkeiten. Entwicklung vom Kindergarten bis zur vierten Grundschulklasse. Kindheit und Entwicklung 2006;15:55-67.

Gasteiger-Klicpera B, Klicpera C, Schabmann A. Der Zusammenhang zwischen Lese-, Rechtschreib- und Verhaltensschwierigkeiten. Kindheit und Entwicklung 2006;15(1):55-67.

Gittelman R, Klein DF, Feingold I. Children with reading disorders. II. Effects of methylphenidate in combination with reading remediation. Journal of Child Psychology and Psychiatry 1983;24(2):193-212.

Goldston DB, Walsh A, Mayfield Arnold E, Reboussin B, Sergent Daniel S, Erkanli A, et al. Reading problems, psychiatric disorders, and functional impairment from mid- to late adolescence. Journal of the American Academy of Child and Adolescent Psychiatry 2007;46(1):25-32.

Häßler F, Tilch P. Piracetam in der Kinder- und Jugendpsychiatrie. Pädiatrie und ihre Grenzgebiete 1996;35:1930.

Hinshaw SP. Externalizing behavior problems and academic underachievement in childhood and adolescence: causal relationships and underlying mechanisms. Psychological Bulletin 1992;111(1):127-55.

Knox E, Conti-Ramsden G. Bullying risks of 11-year-old children with specific language impairment (SLI): Does school placement matter? International Journal of Language and Communication Disorders 2003;38:112.

Limm H, Suchodoletz Wv. Belastungserleben von Müttern sprachentwicklungsgestörter Kinder. Praxis der Kinderpsychologie und Kinderpsychiatrie 1998;47:541-551.

Maughan B. Annotation: Long-term outcomes of developmental reading problems. Journal of Child Psychology and Psychiatry 1995;36:357-371.

Mawhood L, Howlin P, Rutter M. Autism and developmental receptive language disorder - a comparative follow-up in early adult life. I: Cognitive and language outcomes. Journal of Child Psychology and Psychiatry 2000;41(5):547-59.

Meyer-Probst B. Enzephalopathie-Fragebogen - E-F. In. Berlin-Ost: Psychodiagnostisches Zentrum HumboldtUniversität; 1983. 
Milich R, McAninch CB, Harris MJ. Effects of stigmatizing information on children's peer relations: Believing is seeing. School Psychology Review 1992;21:400-409.

Naylor MW, Staskowski M, Kenney MC, Kind CA. Language disorders and learning disabilities in school-refusing adolescents. Journal of the American Academy of Child and Adolescent Psychiatry 1994;33:1331-1337.

Norvilitis JM, Scime M, Lee JS. Courtesy stigma in mothers of children with Attention-Deficit/Hyperactivity Disorder: A preliminary investigation. Journal of Attention Disorders 2002;6:61-68.

Noterdaeme M, Amorosa H. Verhaltensauffälligkeiten bei sprachentwicklungsgestörten Kindern. Monatsschrift der Kinderheilkunde 1998;146:931-937.

Spitczok von Brisinski I. Bullying/Mobbing in der Schule und in der stationären Behandlung unter Berücksichtigung von ADS und Asperger-Syndrom. Forum der Kinder- und Jugendpsychiatrie und Psychotherapie 2005;15:83-115.

Strehlow U. Langfristige Perspektiven von Kindern mit Lese-Rechtschreibstörungen. In: Suchodoletz Wv, (Hrsg.). Welche Chancen haben Kinder mit Entwicklungsstörungen? Göttingen: Hogrefe; 2004. p. 201-218.

Suchodoletz Wv, Keiner T. Psychiatrische Aspekte bei sprachgestörten Kindern. pädiatrische praxis 1998;54: 395-402.

Suchodoletz Wv, Machery G. Stigmatisierung sprachgestörtert Kinder aus Sicht der Eltern. Praxis der Kinderpsychologie und Kinderpsychiatrie 2006;55:711-723.

Suchodoletz Wv. Zur Prognose von Kindern mit umschriebenen Sprachentwicklungsstörungen. In: Suchodoletz Wv, (Hrsg.). Welche Chancen haben Kinder mit Entwicklungsstörungen? Göttingen: Hogrefe; 2004. p. 155-199.

Warnke A, Amorosa H, Aster Mv, Oehler K, Strehlow U, Niebergall G, et al. Umschriebene Entwicklungsstörungen schulischer Fertigkeiten (F81). In: Deutsche Gesellschaft, Bundesarbeitsgemeinschaft leitender Klinikärzte und Berufsverband der Ärzte für Kinder- und Jugendpsychiatrie und Psychotherapie, (Hrsg.). Leitlinien zu Diagnostik und Therapie von psychischen Störungen im Säuglings-, Kindes- und Jugendalter. 3. Aufl. ed. Köln: Deutscher Ärzte-Verlag; 2007. p. 207-224.

Weltgesundheitsorganisation (WHO). Internationale Klassifikation psychischer Störungen. ICD-10 Kapitel V (F). Klinisch-diagnostische Leitlinien. Bern: Hans Huber; 2005.

Willcutt EG, Pennington BF. Psychiatric comorbidity in children and adolescents with reading disability. Journal of Child Psychology and Psychiatry and Allied Disciplines 2000;41:1039-1048.

Williams S, McGee R. Reading attainment and juvenile delinquency. Journal of Child Psychology and Psychiatry 1994;35(3):441-59.

Wilsher-Colin R, Taylor EA. Piracetam in developmental reading disorders: A review. European Child \& Adolescent Psychiatry 1994;3:59-71. 